\title{
ANALYSIS OF TIME-VARYING PSORIASIS LESION IMAGE PATTERNS
}

\author{
G. Maletti, B. Ersbøll, A. Nielsen, D. Delgado Gómez \\ Technical University of Denmark \\ Informatics and Mathematical Modelling \\ Section for Image Analysis and Computer Graphics \\ DK-2800 Kgs. Lyngby, Denmark
}

\begin{abstract}
The Multi-variate Alteration Detection Transform is applied to pairs of within and between time varying registered psoriasis image patterns. Color band contribution to the variates explaining maximal change is analyzed.
\end{abstract}

\section{INTRODUCTION}

We use a set of registered psoriasis lesion patterns segmented from a set of images taken at the Gentofte Hospital, Denmark during four pilot sessions with three invited patients. On those occasions, three lesions per patient were captured with an integrated sphere [1] five times during each session, along four weeks. The images were labelled with four characters, indicating patient $(1,2,3)$, lesion $(A, B, C)$, session (a, b, c, d) and capture $(1,2,3,4,5)$ respectively. From a subset of these images, lesion patterns were segmented and registered within and between sessions using the schemes reported in [6]. Basically, the registration scheme is composed of two stages. In the first stage, global rotation and translation effects of assumed segmented patterns of equally scaled psoriasis lesions are removed. In the second stage, it is assumed that there are no local rotation effects and, therefore, only local translation effects are removed.

Previous work done in registration of lesions in skin images can be found in [9]. In this case, an algorithm considers each lesion as a point. The input to the scheme are multiple lesions skin images. Two initial matches, called the "baseline", are required in order to

G.M.M.'s current address: The Royal Veterinary and Agricultural University, Department of Agricultural Sciences, Section for Agrotechnology, Højbakkegaard Allè 2, DK-2630 Taastrup, Denmark. To the SITE Project funded by a grant from the Danish Technical Research Foundation (Project Number STVF 5600-0123) for supporting the present work. To the dermatologists Lone Skov and Bo Bang of the Gentofte Hospital of Denmark and to the anonymous patients, for their collaboration during the image acquisition sessions. find the remaining correspondent points. Related work in dermatological images can be found in [3], [2], [11].

To evaluate lesion changes between sessions, physicians make scores of a four-variable set: redness, scaling, thickness and body area covered by the lesions. Manual scoring depends strongly on the dermatologist, who can show criteria variations along sessions due to the huge amount of patients and lesions observed during each working day.

The main objective of the present work is to analyze changes in time [8], [10], [4] from a statistical point of view of the registered data sets mentioned. A suitable statistical approach for analyzing changes in time in a multi-variable fashion is the Multi-variate Alteration Detection Transform (M.A.D.) [8]. It transforms two sets of multi-variate observations into a difference between two linear combinations of the original variables explaining maximal change in all variables simultaneously. The magnitude of the lesion changes in time could be shown using the absolute value of the first M.A.D. component. Alternatively, the color band contribution to the change could be calculated.

The present work is composed of the following sections. First, general aspects of the M.A.D. Transform are described. Then, an experiment to compute the color band contribution in the lesions change in time is designed. A section of results and discussion follows.

\section{M.A.D. TRANSFORM}

Following [8], let $X_{1}$ and $X_{2}$ be two sets of Gaussian distributed variables of dimension $m_{1}$ and $m_{2}$ respectively, with $m_{1} \leq m_{2}, E\left\{X_{1}\right\}=E\left\{X_{2}\right\}=0$ transformed with the coefficients from a standard canonical correlation analysis $\vec{a}_{1}$ and $\vec{a}_{2}$ in

$$
\begin{aligned}
& Y_{1 i}=\vec{a}_{1 i}^{T} X_{1}, i=1, \ldots, m_{1} \\
& Y_{2 i}=\vec{a}_{2 i}^{T} X_{2}, i=1, \ldots, m_{2},
\end{aligned}
$$

positively correlated and with unit variance, such that the variance of their difference is maximized. Note 
that:

$$
\begin{gathered}
\operatorname{Var}\left[\vec{a}_{1}^{T} X_{1}-\vec{a}_{2}^{T} X_{2}\right]= \\
=2\left(1-\operatorname{Corr}\left[\vec{a}_{1}^{T} X_{1}, \vec{a}_{2}^{T} X_{2}\right]\right)
\end{gathered}
$$

The M.A.D. Transform consists of the variates to get when the corresponding canonical variates are subtracted in reverse order, which means that the $m_{1}^{\text {th }}$ difference shows maximum variance among such variables, and the $\left(m_{1}-j\right)^{t h}$ difference shows maximum variance subject to the constraint that this difference is not correlated with the previous $j$ ones. The M.A.D. variates are invariant to linear and affine scaling, which is not the case of the Principal Components. The dispersion matrix of the M.A.D. variates is

$$
D\left\{\vec{a}_{1}^{T} X_{1}-\vec{a}_{2}^{T} X_{2}\right\}=2(I-R)
$$

where $I$ is the $m_{1} \times m_{1}$ unit matrix and $R$ is the $m_{1} \times m_{1}$ matrix containing the sorted canonical correlations on the diagonal and zeros off the diagonal.

\section{RESULTS AND DISCUSSION}

The contribution of each single color band in showing the lesion change in a period of one week is analyzed here. Results are confronted with the original image data. Lesion changes between sessions are also described using the absolute value of the first M.A.D. component.

Following with the notation used in previous work ([6], [7]), the groups of images corresponding to a given patient and lesion are here called "case (patient, lesion)". The cases registered in $[6]$ are $(1, A),(1, B)$, $(1, C),(2, A),(2, B)$ and $(3, C)$.

The set of 108 registered images produced by the registration scheme allowed the construction of 377 pairs $^{1}$ of registered lesions with the same time increment of one week between them. For each image of a given patient and lesion, each one of the images of the next session were associated. For each pair of synthetical images constructed in the way mentioned, the M.A.D. transform was applied [5]. The M.A.D. Transform was only applied to the pixels belonging to the lesion.

Some outputs produced during the experiment mentioned before are shown in Figures 1 and 2. For each case, registered image patterns, corresponding to the first image of each session, and the absolute first M.A.D. components of pairs of those patterns with a time separation of one week can be seen. In order to show the magnitude of the change, the absolute value of the first M.A.D. component has been computed. Light colors

\footnotetext{
${ }^{1}$ For each lesion the number of pairs is $(1, A)=25 \times 3 ;(1, B)=$ $9 \times 3 ;(1, C)=25 \times 2 ;(2, A)=25 \times 3 ;(2, B)=25 \times 3$ and $(2, C)=25 \times 3$ respectively.
}

in the figures corresponding to the absolute value of the first M.A.D. components imply more change in the lesion; dark colors imply the opposite situation. For instance, for the case $(1, A)$, in the upper part of the figure we have four registered patterns, corresponding to the first, second, third and fourth week. In the lower part of the same figure, we have from the left to the right, the absolute value of the first M.A.D. component from the first to the second week, from the second to the third week, and, from the third to the fourth week respectively. The figures corresponding to the remaining cases are presented in the same way. Some comments regarding to visual examination of the results follow.
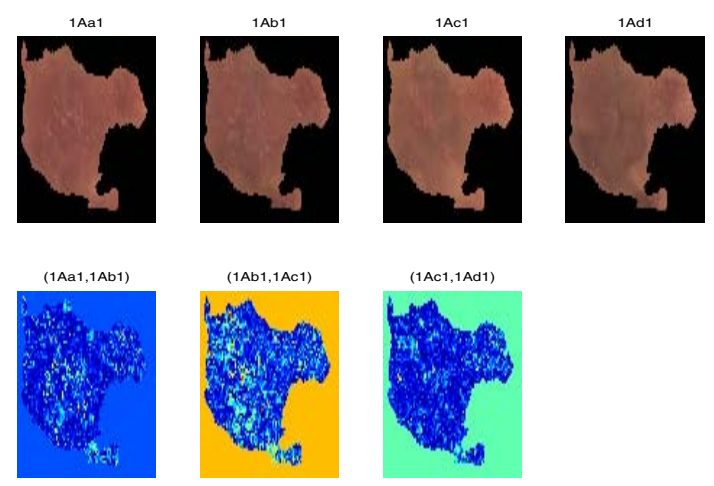

Fig. 1. Registered Images for the case $(1, A)$ and the First M.A.D. components per week.
1Ca1
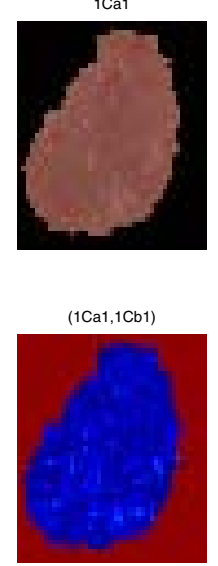

$1 \mathrm{Cb} 1$
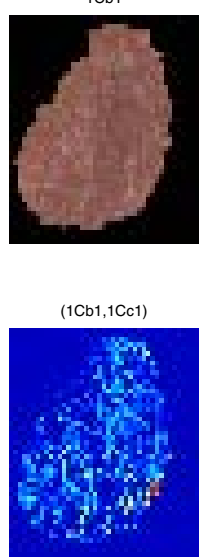

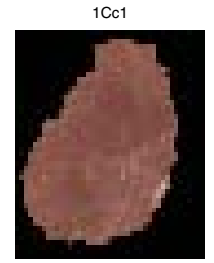

Fig. 2. Registered Images for the case $(1, C)$ and the First M.A.D. components per week.

For the case $(1, A)$, it can be seen that most of the 
change from the first to the second week occurs in the central part of the lesion. Afterwards, from the second to the third week, it spreads out. From the third to the fourth week, most of the change occurs near the borders. The same behavior can be described for the case $(1, B)$ and for the first three weeks of the case $(1, C)$. In the upper part of the first M.A.D. component from the first to the second week of the case $(1, B)$ a large change is indicated. Looking at the original images, it seems that this aspect could be indicating different illumination conditions [7] between the captures, more than lesion changes. The reader is reminded that the mentioned cases do correspond to the same patient. For the case $(2, A)$ an interesting peak can be observed in the absolute value of the first M.A.D. component from the first to the second week. This is not easily appreciable in the original images. Clearly the M.A.D. transform is able to show a change that cannot be seen in the original images. For the case $(2, B)$, it can again be observed that the change starts in the center of the lesion and in the next week it spreads out. It was mentioned in [6], that the registration algorithm gets a bit confused in the presence of hair. Hair displacements between sessions can not easily be seen in the absolute value first M.A.D. component. This may be because of the quality of the registration output itself. For the case $(3, C)$, the presence of scaling in the second week is clearly indicated by large absolute values of the first M.A.D. component, from the first to the second week, and, from the second to the third week. However, peaks appearing in the absolute value of the first M.A.D. component from the third to the fourth week are clearly skin displacements attained after folding the elbow. In general, it could be said that the absolute value of the first M.A.D. component shows in a very clear manner, the appearing and disappearing of scaling.

Tables 1 and 2 show the general obtained for the sets of registered images. Each single cell value $E_{(b, m, p, l)}$ in Table 1 is the average absolute correlation value between the $b-t h$ color bands and the $m-t h$ MAD components of all pairs of images of the $l-t h$ lesion of the $p-t h$ patient. In general, for each case $(p, l)$, it is computed in the following way:

$E_{b, m}=E\left[\frac{1}{2}\left(\left|\operatorname{Corr}\left\{X_{1, b}, M A D_{m}\right\}\right|+\left|\rho\left\{X_{2, b}, M A D_{m}\right\}\right|\right)\right]$

where Corr indicates the correlation coefficient. Similarly, each single cell $\sigma_{b, m, p, l}$ value in Table 2 is the standard deviation $\sigma$ of the value between brackets in Equation 5.

Excluding from Table 1 the case for which the registered lesion patterns are partially covered by hair $(\mathrm{Pa}-$ tient 2, Lesion B), we can observe that, in general, most
Table 1. Average Absolute Correlation Values per Lesion of $R G B$ registered lesion data with their respective M.A.D. Components

\begin{tabular}{|c|ccc|}
\hline Case & (R,MAD1) & (G,MAD1) & (B,MAD1) \\
\hline$(1, \mathrm{~A})$ & 0.239941 & 0.312891 & 0.479555 \\
$(1, \mathrm{~B})$ & 0.194602 & 0.342770 & 0.404496 \\
$(1, \mathrm{C})$ & 0.502525 & 0.582041 & 0.602087 \\
$(2, \mathrm{~A})$ & 0.273979 & 0.444085 & 0.298466 \\
$(2, \mathrm{~B})$ & 0.232567 & 0.325108 & 0.154075 \\
$(3, \mathrm{C})$ & 0.399863 & 0.536893 & 0.529572 \\
\hline Case & (R,MAD2) & (G,MAD2) & (B,MAD2) \\
\hline (1,A) & 0.071068 & 0.267727 & 0.141211 \\
$(1, \mathrm{~B})$ & 0.086706 & 0.122383 & 0.123006 \\
$(1, \mathrm{C})$ & 0.211631 & 0.176254 & 0.224510 \\
$(2, \mathrm{~A})$ & 0.185631 & 0.198860 & 0.123149 \\
$(2, \mathrm{~B})$ & 0.123701 & 0.157495 & 0.116539 \\
$(3, \mathrm{C})$ & 0.314972 & 0.258697 & 0.225947 \\
\hline Case & $(\mathrm{R}, \mathrm{MAD} 3)$ & $(\mathrm{G}, \mathrm{MAD} 3)$ & $(\mathrm{B}, \mathrm{MAD} 3)$ \\
\hline$(1, \mathrm{~A})$ & 0.246138 & 0.150544 & 0.097587 \\
$(1, \mathrm{~B})$ & 0.324035 & 0.278036 & 0.255027 \\
$(1, \mathrm{C})$ & 0.254546 & 0.171539 & 0.071888 \\
$(2, \mathrm{~A})$ & 0.246606 & 0.161208 & 0.262824 \\
$(2, \mathrm{~B})$ & 0.302258 & 0.267418 & 0.318841 \\
$(3, \mathrm{C})$ & 0.212062 & 0.127252 & 0.186059 \\
\hline
\end{tabular}

Table 2. Standard Deviation of the Absolute Correlation Values per Lesion of the $R G B$ registered lesion data with the M.A.D. Components

\begin{tabular}{|c|ccc|}
\hline Case & (R,MAD1) & (G,MAD1) & (B,MAD1) \\
\hline$(1, \mathrm{~A})$ & 0.082760 & 0.088492 & 0.076644 \\
$(1, \mathrm{~B})$ & 0.062977 & 0.088879 & 0.101755 \\
$(1, \mathrm{C})$ & 0.071854 & 0.051745 & 0.044919 \\
$(2, \mathrm{~A})$ & 0.145003 & 0.130561 & 0.081781 \\
$(2, \mathrm{~B})$ & 0.043526 & 0.078260 & 0.060818 \\
$(3, \mathrm{C})$ & 0.120938 & 0.129143 & 0.099749 \\
\hline Case & $(\mathrm{R}, \mathrm{MAD} 2)$ & $(\mathrm{G}, \mathrm{MAD} 2)$ & $(\mathrm{B}, \mathrm{MAD} 2)$ \\
\hline$(1, \mathrm{~A})$ & 0.047430 & 0.051808 & 0.072253 \\
$(1, \mathrm{~B})$ & 0.042134 & 0.102374 & 0.107343 \\
$(1, \mathrm{C})$ & 0.088767 & 0.095334 & 0.078513 \\
$(2, \mathrm{~A})$ & 0.103476 & 0.125162 & 0.076504 \\
$(2, \mathrm{~B})$ & 0.055127 & 0.103429 & 0.061193 \\
$(3, \mathrm{C})$ & 0.167413 & 0.165183 & 0.135105 \\
\hline Case & $(\mathrm{R}, \mathrm{MAD} 3)$ & $(\mathrm{G}, \mathrm{MAD} 3)$ & $(\mathrm{B}, \mathrm{MAD} 3)$ \\
\hline$(1, \mathrm{~A})$ & 0.021716 & 0.022367 & 0.038063 \\
$(1, \mathrm{~B})$ & 0.074971 & 0.062505 & 0.061780 \\
$(1, \mathrm{C})$ & 0.052423 & 0.057971 & 0.050960 \\
$(2, \mathrm{~A})$ & 0.023292 & 0.017698 & 0.027054 \\
$(2, \mathrm{~B})$ & 0.027727 & 0.028042 & 0.025397 \\
$(3, \mathrm{C})$ & 0.069320 & 0.051744 & 0.049821 \\
\hline
\end{tabular}




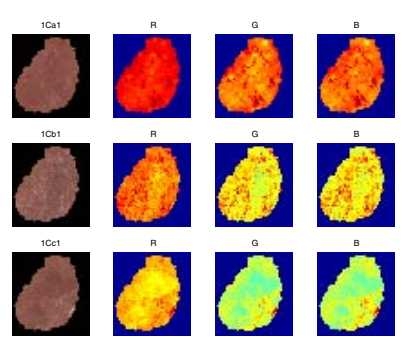

Fig. 3. $R G B$ bands of registered images of the case $(1, C)$

of the contribution in the first M.A.D. component is given either by the green or the blue band. These results can be verified, by visual examination of the registered lesions image patterns decomposed in its $R G B$ bands. See, for instance, Figure 3.

An aspect that was not considered here, because it is not the objective of the present work, is that the M.A.D. transform, as it was conceived in [8], requires positive correlation of the canonical variables. The cases for which the canonical variables are not positively correlated ${ }^{2}$, could be interesting to analyze. This is referred to the meaning of the output of the difference of negatively correlated canonical variables. If the M.A.D. Transform is extended to all the orientations of the two sets of eigenvectors by flipping the eigenvectors values of the original variables, a set of four possible solutions is produced.

\section{CONCLUSIONS}

The small number of lesions evaluated is not enough to make general conclusions about the disease behavior. However, for the cases analyzed the following can be mentioned. Looking at the absolute value of the first M.A.D. component of pairs of registered lesion patterns separated by a period of one week, it can be observed that in the beginning, most of the change occurs within the lesion, while in the next week it spreads out and is distributed on the whole lesion. In some cases it has been observed, that in the lasts sessions, the change occurs near the lesion borders.

Regarding the contribution of each single color band to the first M.A.D. component, it has been shown that, in general, it is either the green or the blue band, and not the red band which contributes most in showing the change.

\footnotetext{
${ }^{2}$ In the psoriasis lesions data set there cases for which the correlation between pairs of images is negative. See for instance, in Tables 6,8 and 10 of [6] the values corresponding to the case $(3, C)$.
}

\section{REFERENCES}

[1] Videometer. http:www.videometer.dk.

[2] D. Chung and G. Sapiro. Segmenting skin lesions with partial differential equation based image processing algorithms. IEEE International Conference on Image Processing, 3(24):404-407, 2000.

[3] G. Hance, S. Umbaugh, R. Moss, and W. Stoecker. Unsupervised color image segmentation. IEEE Engineering in Medicine and Biology, 15(1):104$111,1996$.

[4] B. Horn and B. Schunck. Determining optical flow. Artificial Intelligence, 17:185-204, 1981.

[5] R. Larsen. Maf and other transformations applied to remote sensing. Master's thesis, Institute for Mathematical stastistics and Operations Research (IMSOR), Technical University of Denmark, DTU, 1991. pp. xii $+130+205$.

[6] G. Maletti and B. Ersbøll. A combined alignment and registration scheme of psoriasis lesions images. International Journal in Information Sciences, 2003. Accepted.

[7] G. Maletti and B. Ersbøll. Illumination correction from psoriasis image data. Lecture Notes in Computer Science, 2749:549-556, June 2003. $13^{\text {th }}$ Scandinavian Conference on Image Analysis.

[8] A. Nielsen, K. Conradsen, and J. Simpson. Multivariate alteration detection (m.a.d.) and m.a.f. postprocessing in multispectral, bitemporal image data: New approaches to change detection studies. Remote Sens. Environ., 64:1-19, 1998.

[9] J. Röning and M. Riech. Registration of nevi in successive skin images for early detection of melanoma. $14^{\text {th }}$ International Conference on Pattern Recognition, 1:352-357, 1998.

[10] M. Sonka, V. Hlavac, and R. Boyle. Image Processing, Analysis and Machine Vision. Brooks Cole Publishing Company, Pacific Grove, CA 93950, USA., 2nd edition, 1999.

[11] M. Soriano, B. Martinkauppi, S. Huovinen, and M. Laaksonen. Skin detection in video under changing illumination conditions. $15^{\text {th }}$ International Conference on Pattern Recognition, 1:839$842,2000$. 